\title{
Non-supervised method for early forest fire detection and rapid mapping
}

Tomàs Artés, Roberto Boca, Giorgio Liberta, Jesús SanMiguel

Tomàs Artés, Roberto Boca, Giorgio Liberta, Jesús San-Miguel, "Nonsupervised method for early forest fire detection and rapid mapping," Proc. SPIE 10444, Fifth International Conference on Remote Sensing and Geoinformation of the Environment (RSCy2017), 104440R (6 September 2017); doi: 10.1117/12.2280714

SPIE Event: Fifth International Conference on Remote Sensing and Geoinformation of the Environment (RSCy2017), 2017, Paphos, Cyprus 


\title{
Non-supervised method for early forest fire detection and rapid mapping
}

\author{
Tomàs Artés*a, Roberto Boca ${ }^{\mathrm{b}}$, Giorgio Liberta’a, Jesús San-Miguel, ${ }^{\mathrm{a} J o i n t ~ R e s e a r c h ~ C e n t r e, ~ V i a ~}$ \\ Enrico Fermi 1 Ispra, Italy
}

\begin{abstract}
Natural hazards are a challenge for the society. Scientific community efforts have been severely increased assessing tasks about prevention and damage mitigation. The most important points to minimize natural hazard damages are monitoring and prevention. This work focuses particularly on forest fires. This phenomenon depends on small-scale factors and fire behavior is strongly related to the local weather. Forest fire spread forecast is a complex task because of the scale of the phenomena, the input data uncertainty and time constraints in forest fire monitoring. Forest fire simulators have been improved, including some calibration techniques avoiding data uncertainty and taking into account complex factors as the atmosphere. Such techniques increase dramatically the computational cost in a context where the available time to provide a forecast is a hard constraint. Furthermore, an early mapping of the fire becomes crucial to assess it. In this work, a nonsupervised method for forest fire early detection and mapping is proposed. As main sources, the method uses daily thermal anomalies from MODIS and VIIRS combined with land cover map to identify and monitor forest fires with very few resources. This method relies on a clustering technique (DBSCAN algorithm) and on filtering thermal anomalies to detect the forest fires. In addition, a concave hull (alpha shape algorithm) is applied to obtain rapid mapping of the fire area (very coarse accuracy mapping). Therefore, the method leads to a potential use for high-resolution forest fire rapid mapping based on satellite imagery using the extent of each early fire detection. It shows the way to an automatic rapid mapping of the fire at high resolution processing as few data as possible.
\end{abstract}

Keywords: Forest fire detection, rapid mapping, clustering, fire scar, DBSCAN, burnt area, fire behavior

\section{INTRODUCTION}

Forest fire is a natural phenomenon, which is part of the ecosystem cycle. However, human activities are changing the frequency of the fires occurrences and the burnt area as well. Although every country have highly qualified firefighters to stop, reduce and assess the fires, the number of fires for a given area could be high enough to collapse the resources available. In fact, countries implemented mechanisms to share resources between them trying to avoid the severe damages during intense seasons. This seasonal behavior of the fire could be so extreme that resources are eventually shared even between transoceanic countries like the forest fires that took place in Chile on 2017. Frequently, the most common cause of forest fires is human action instead of natural phenomena like lightings. The occurrence of fires is not easy to predict even during some seasons. Fire occurrences and burnt areas could be very different in close zones that belongs to different countries.

Prevention tasks are carried out to avoid severe damages. Nevertheless, such pre-event works are expensive and could not be feasible in some cases. These previously mentioned pre-event tasks may require legislative implementations since some forest areas are private. Also, several works deal with forest treatment to avoid high fire severity like thinning vegetation [1] [2] in order to reduce the potential fuel. The prevention must be done when there is a previous knowledge that leads to a high probability of forest fire in a specific zone.

*tomas.artes-vivancos@ec.europa.eu; phone +39 0332-783930; http://forest.jrc.ec.europa.eu/effis/about-effis/

Fifth International Conf. on Remote Sensing and Geoinformation of the Environment (RSCy2017), edited by Kyriacos Themistocleous, et al., Proc. of SPIE 10444, 104440R · (c) The Authors.

Published under a Creative Commons Attribution CC BY 3.0 License $\cdot$ doi: 10.1117/12.2211588 
In addition, there is a big effort in research community trying to model the forest fire behavior. Several models have been developed and implemented in software simulators. These simulators could help to decision-making teams to forecast the fire behavior. The main drawbacks of such models are input data uncertainty, computing complexity and initialization. When the fire starts is hard to know its current fire front, the moistures of the fuels and the weather conditions at high resolution. Several approaches using data assimilation have been carried out to avoid data uncertainty [3] [4] [5]. Forest fire modelling is computed at high spatial resolution scale, in the order of meters. When using complex models that take into account as many parameters as possible, including the interaction of the atmosphere, the computing cost and the initialization becomes a not easy task [6]. Several research communities try to simplify the problem and provide tools to assess the fire behavior in real-time [7] [8].

Finally, post-event tasks are very costly and should be done as fast as possible. Fire severity could hardly damage the vegetation and the soil. Then, when the fire severity is high enough, the fire causes changes in the soil composition and consequently in its behavior regarding the water flows. Consequently, vegetation regeneration is very slow and the topography as it was is lost. Therefore, it is crucial to focus the efforts in specific areas where is decisive to take measures to maintain the soil and achieve a good regeneration of the vegetation.

Aiming to improve the efficiency of the tasks during the whole forest fire event is convenient to combine about fire behavior, vegetation and soil science. Then, a precise monitoring of the fire becomes crucial. For this reason, this work focuses in fire detection and rapid fire mapping. The paper explains an approach that combines multiple satellite sensors to detect the fire and provide the fire mapping as soon as new data is available.

The first section depicts the algorithm used for fire detection and rapid mapping, followed by a comparison between the fire detection and a human validated fire mapping. The section 2 shows how other sources are used to obtain a more accurate mapping algorithm using satellite images to process only the areas where a fire has been detected. Finally, some preliminary results and conclusions are exposed.

\section{FIRE DETECTION AND RAPID MAPPING}

\subsection{Algorithm}

One of the remote sensing products that provide information in near real-time is the Thermal Anomalies from NASA [9] [10]. This product has been validated, tested and finally improved by including additional sensors such as Visible Infrared Imaging Radiometer Suite (VIIRS). In fact, this product currently uses Terra and Aqua at $1 \mathrm{~km}$ spatial resolution and VIIRS at 375 meters resolution providing information within three hours of the satellites overpass.

The main goal of the fire detection and rapid mapping is to develop a quick algorithm to identify each fire and to estimate its size and monitor the fire spread. Such method allows to focus on each identified fire an process other sources minimizing the computational cost and increasing a potential parallelism for every case.

Hence, the first step creates clusters with thermal anomalies. Each cluster represents a potential fire. In order to avoid clusters which are false positives, the thermal anomalies are filtered using the CORINNE Land Cover 2012 (CLC2012) [11]. This filtering process is carried out creating an R-Tree with the CLC2012 geometries and it is finally checked with a point-in-polygon procedure for those land covers types that should be taken into account. The R-Tree is created only once and used in the following runs. After that, the clustering process starts. First, a custom K-Nearest Neighbor algorithm was tested [4] [12] and finally replaced by DBSCAN [13] included in Scikit-Learn [14] Python library. A set of labeled groups of thermal anomalies is obtained as output.

Following, a potential burnt area is computed applying the alpha shape algorithm [15] obtaining the convex hull of each set of points. The alpha shape algorithm is applied in a loop and stops when a given set of points creates two different polygons for the convex hull. The loop stop condition is based on the premise that each group should be a single burnt area. At this point, each set of fires have a potential burnt area, an extent of the fire, a percentage of area inside vegetated area, an initial date and time and all the properties of the thermal anomalies associated with the fire. 
A set of classes has been implemented to comprehend an abstract concept of a fire case that allows monitoring and processing the fires in subsequent executions of the fire detection. When the algorithm runs checks the result of the previous execution. Therefore, it takes into account the previous fire detections. Then, the clustering of the current execution adds the points of the previous burnt areas polygons and adds the new thermal anomalies obtained since the last execution. This last step may result in cases where some thermal anomalies are associated to more than one cluster, this means that several fires can joint in a single fire as can be seen in Figure 1.

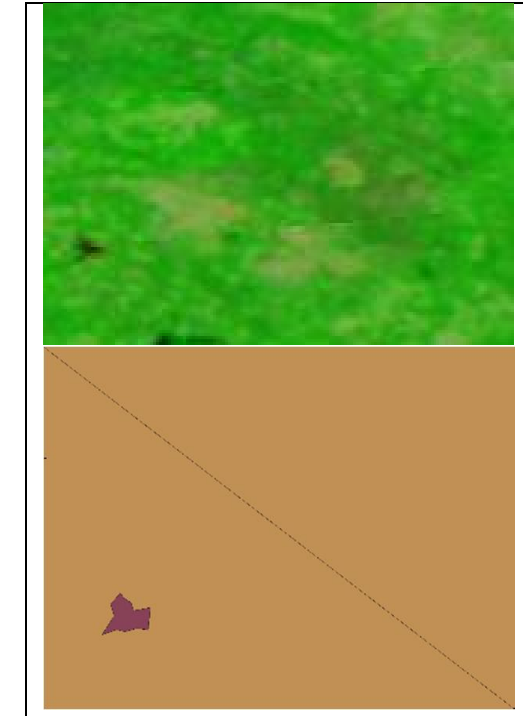

06-08-2016

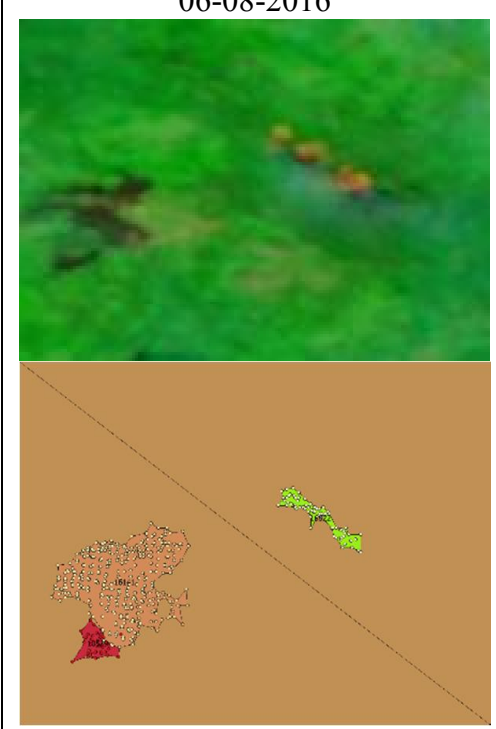

09-08-2016

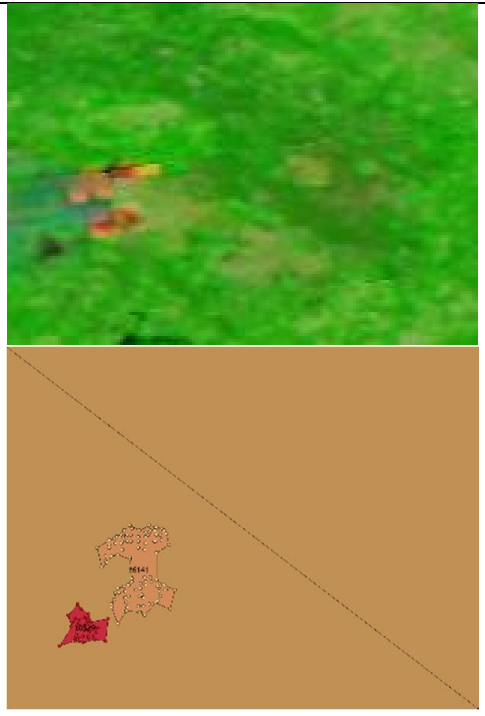

07-08-2016

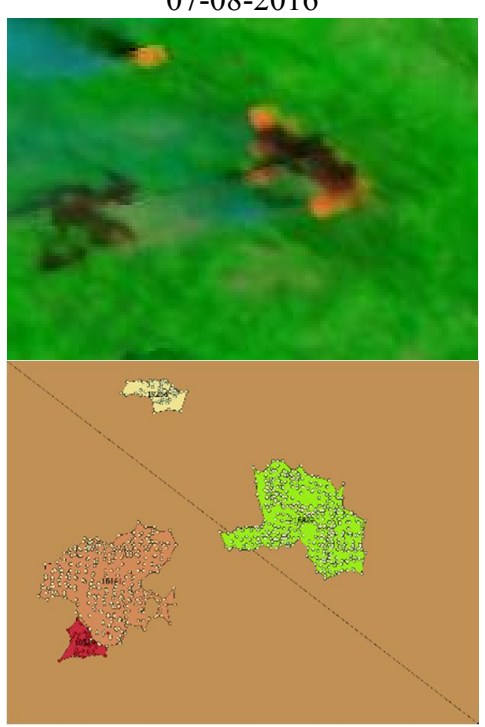

10-08-2016

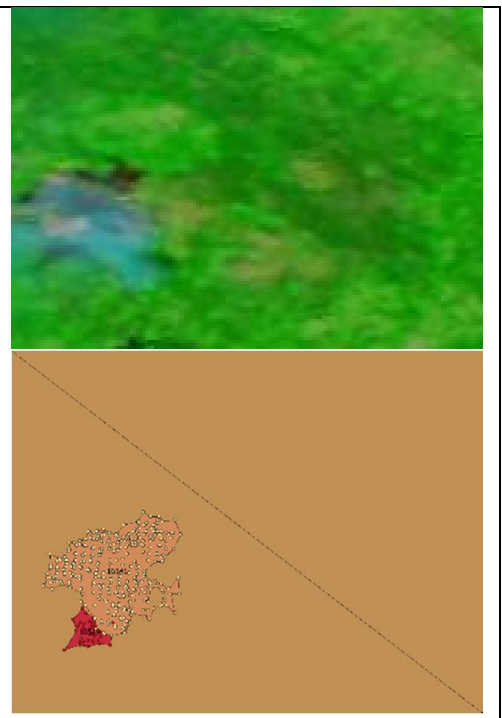

08-08-2016

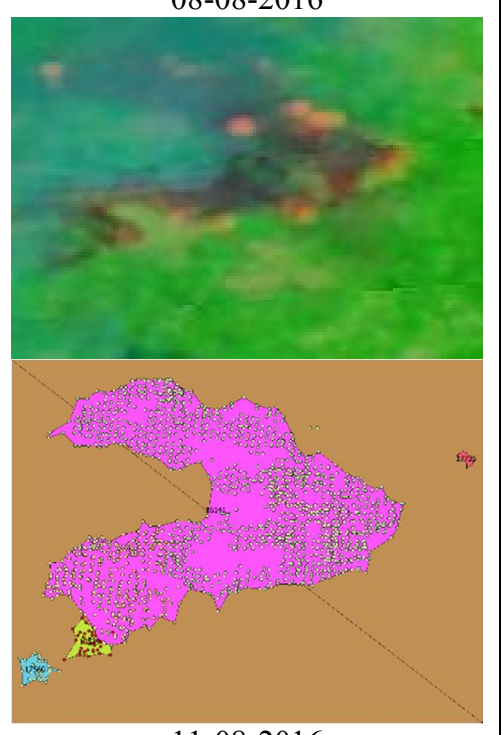

$11-08-2016$

Figure 1. Join fires. Upper images from NPP Suomi corrected reflectance. Below, visualization of the convex hull of the clusters. This sample case took place in Portugal.

In addition, some fire cases are false positives. Even using CLC2012 to filter the thermal anomalies some new industrial areas, volcanoes, etc. could cause false positive fire detections. Aiming to reduce such cases, each fire detection is checked searching for unusual fire behavior. For instance, detections which are rarely active or with a non constant activity during a long period are labeled as potential false positives and each extent is used for an additional filtering 
process for the next fire detection run. See Figure 2. Consequently, some false positives that could be present at some stage may disappear afterwards and used as a filter for later executions.

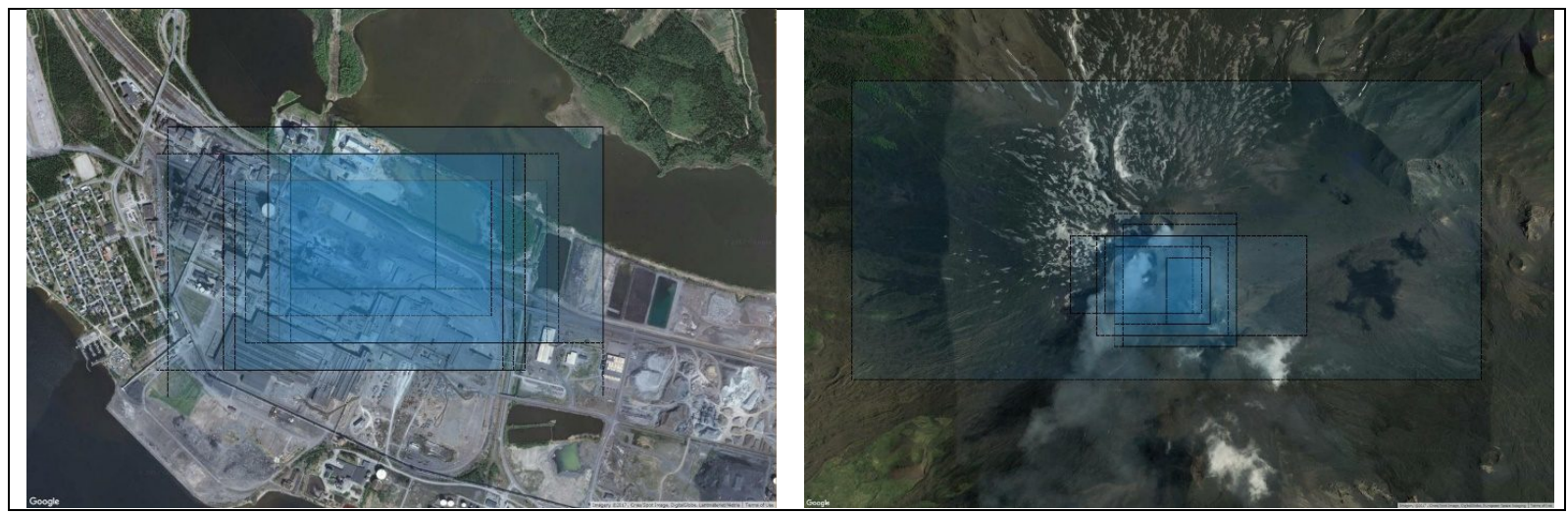

Figure 2. Excluded areas by behavior. Left, industrial area located in Sweden. Right image shows excluded areas in Sicilia, Etna volcanic area.

\subsection{Implementation}

The first development to check the feasibility of the method has been developed in Python using GIS libraries such as GDAL OGR, Shapely or R-Tree. The implementation follows the UML class diagram described in Figure 3 . This scheme allows including new sources and methods for the forest fire mapping process. In addition, the fire collection could be used by other applications inside EFFIS about risk and damage assessment.

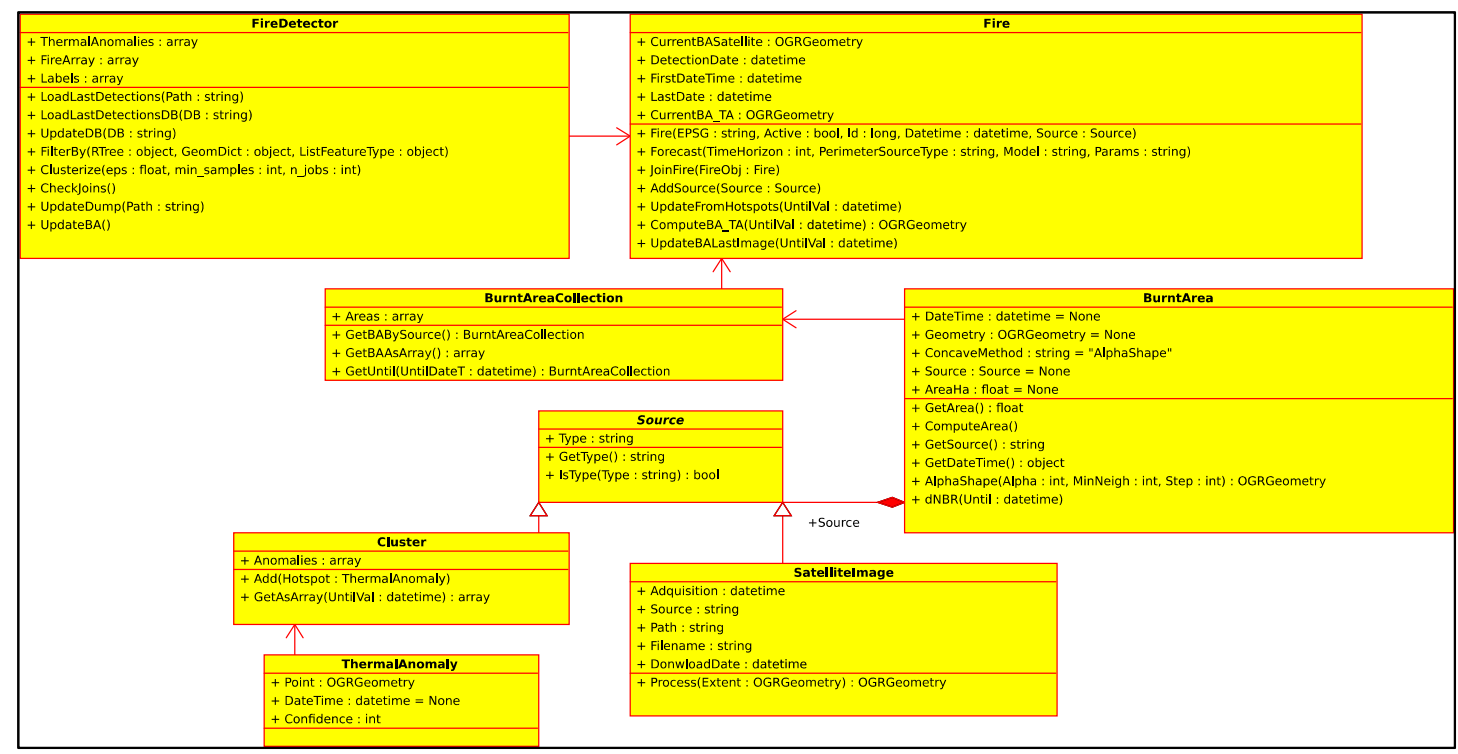

Figure 3. UML class diagram developed for to check the feasibility of the proposed method.

\subsection{Results}

The algorithm do not ensure that every cluster is single fire, since is just a clustering of several points. Nevertheless, its potential is not negligible since could allow much more complex and costly computation focusing on the fire extents. As a first evaluation, the algorithm is run during the fire season of 2016 from date 17/07/2016 to 17/09/2016 using an EPS 
value for the DBSCAN of 0.015 inside the bounding box $((27.1866,-18.8603),(73.19,51.0))$ for Europe. Finally, we compared the results of fire detection and burnt area from the current EFFIS mapping product (with human validation) and the proposed algorithm in this work.

Figure 4 shows the number of fire detections produced by EFFIS and the proposed fire detector. The fire season starts with a similar number of detections. However, there is a difference at the beginning of august. Looking at the daily fire occurrence in Figure 5, the EFFIS mapping product is has a peak of fires that is not seen by the fire detector. It could be several reason for this fact. The current EFFIS service maps the fire as soon as a satellite image from Landsat is available with a good visibility. Thus some fire mapping could be delayed some days until the data sources are good enough to map the fire correctly. In addition, the fire mapping using only the thermal anomalies works well for big fires. That means that little fires in the same area could be mapped as one or even discarded by the low number of thermal anomalies with a low confidence value.

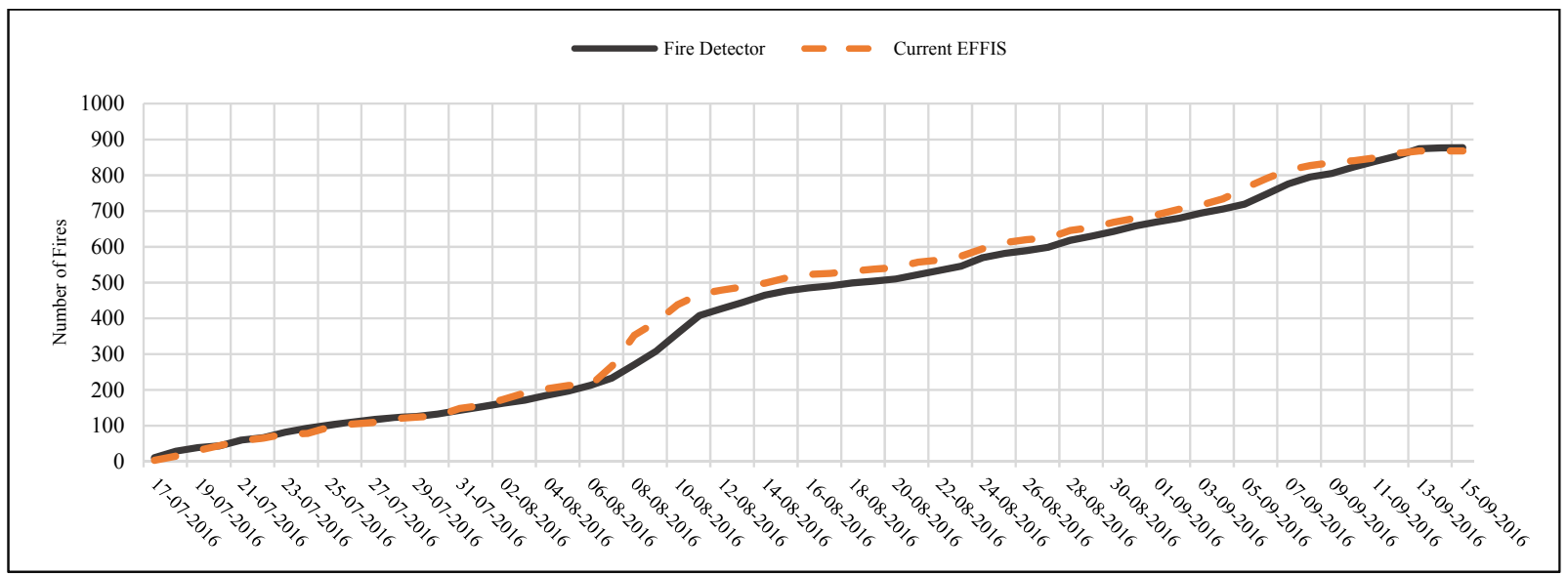

Figure 4. Fire occurrences during the fire season 2016.

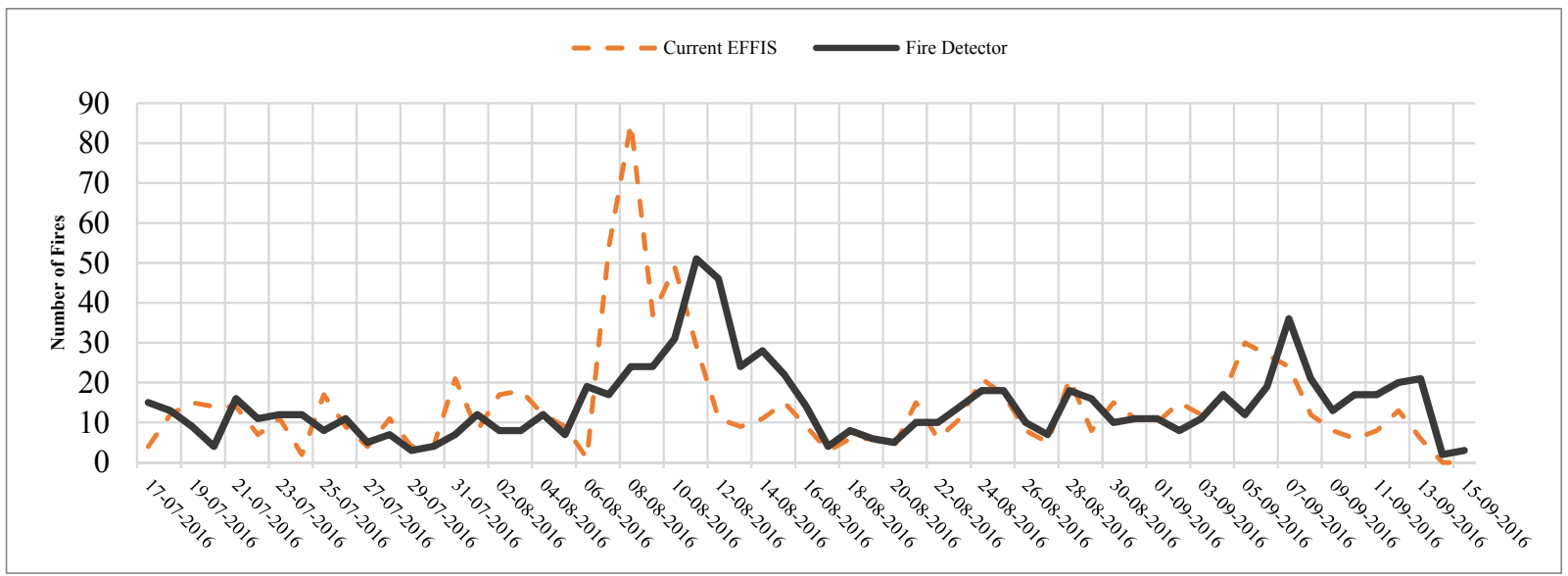

Figure 5. Daily fire occurrences during fire season 2016. 


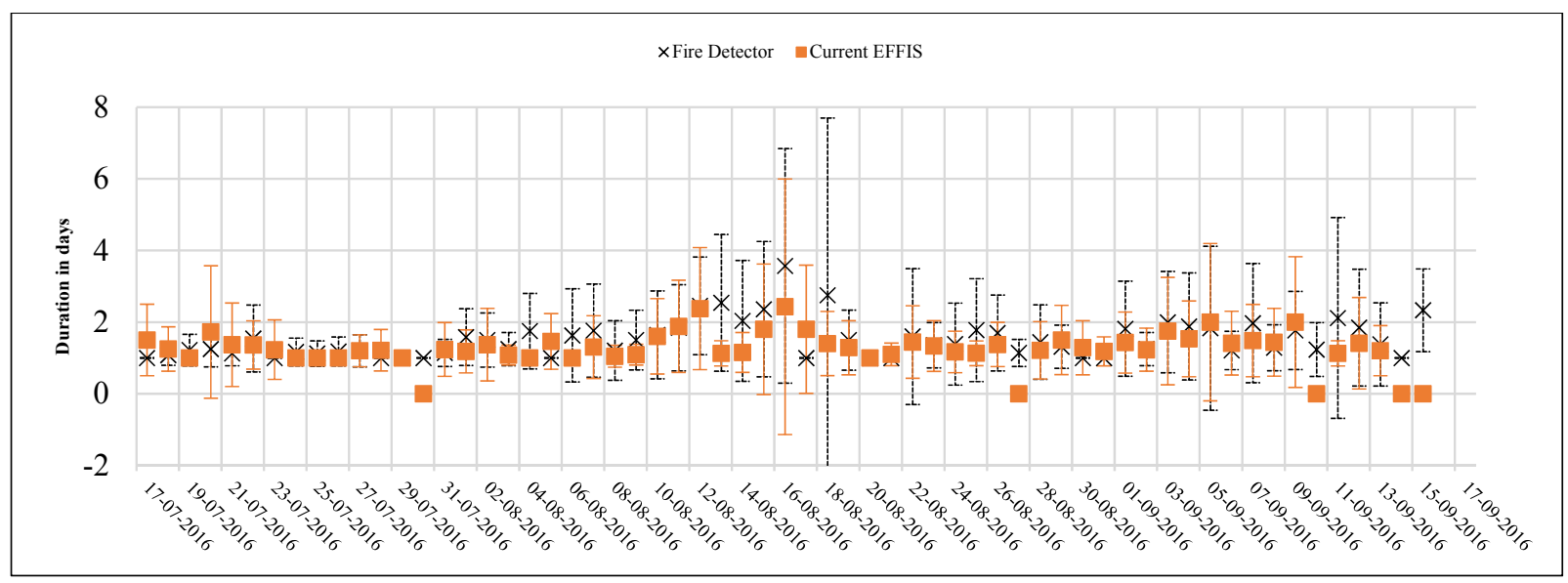

Figure 6. Average fire duration, computed using the fires which are labeled as not active

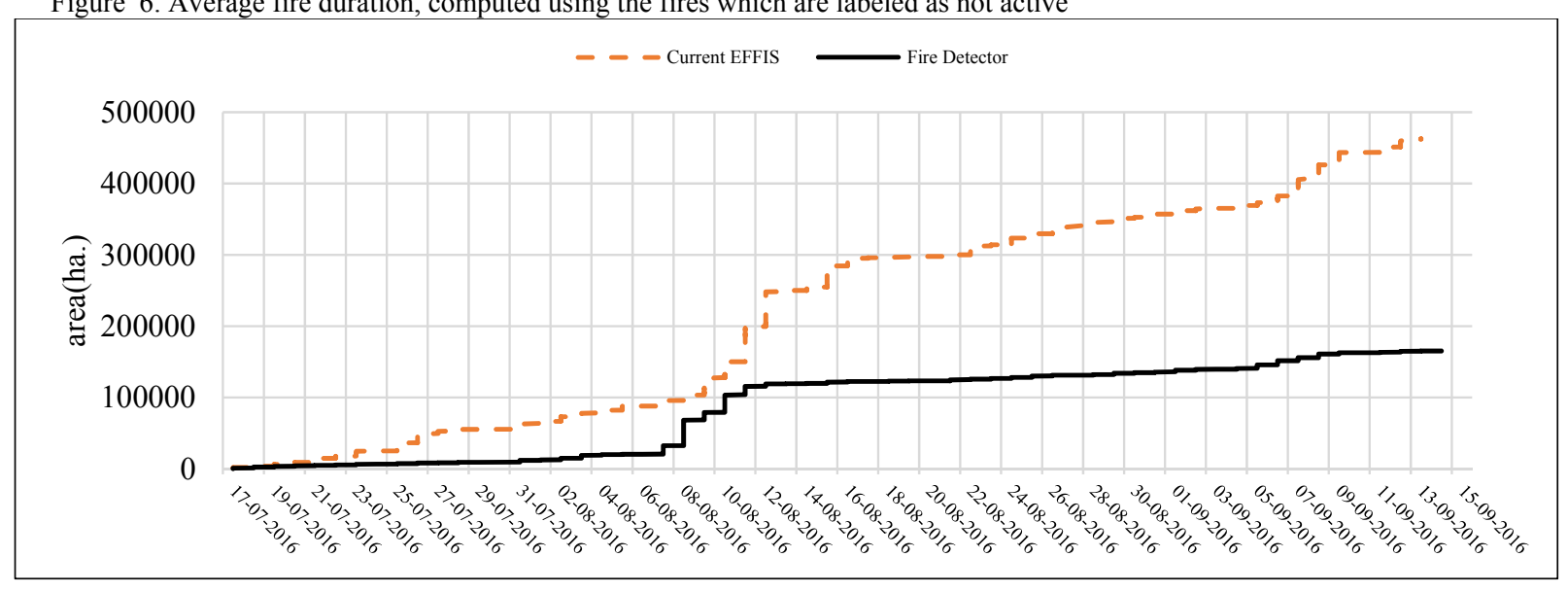

Figure 7. Daily accomulated burnt area.

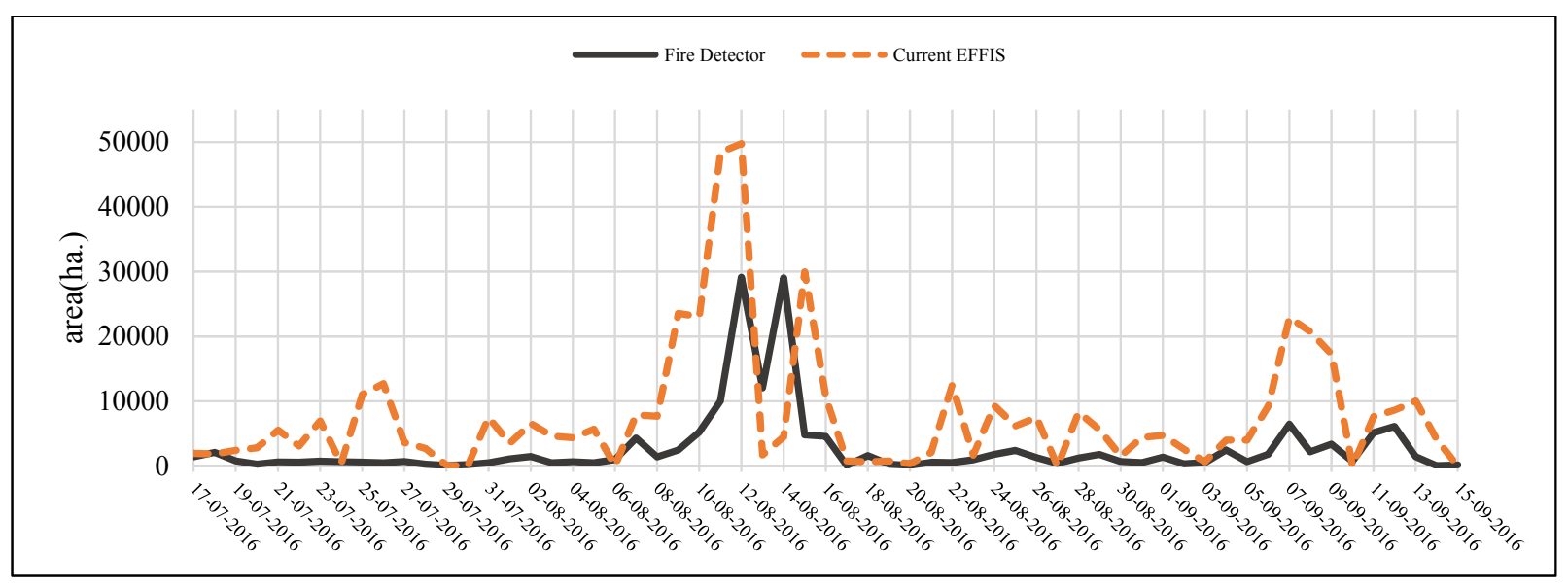

Figure 8. Daily burnt area. 


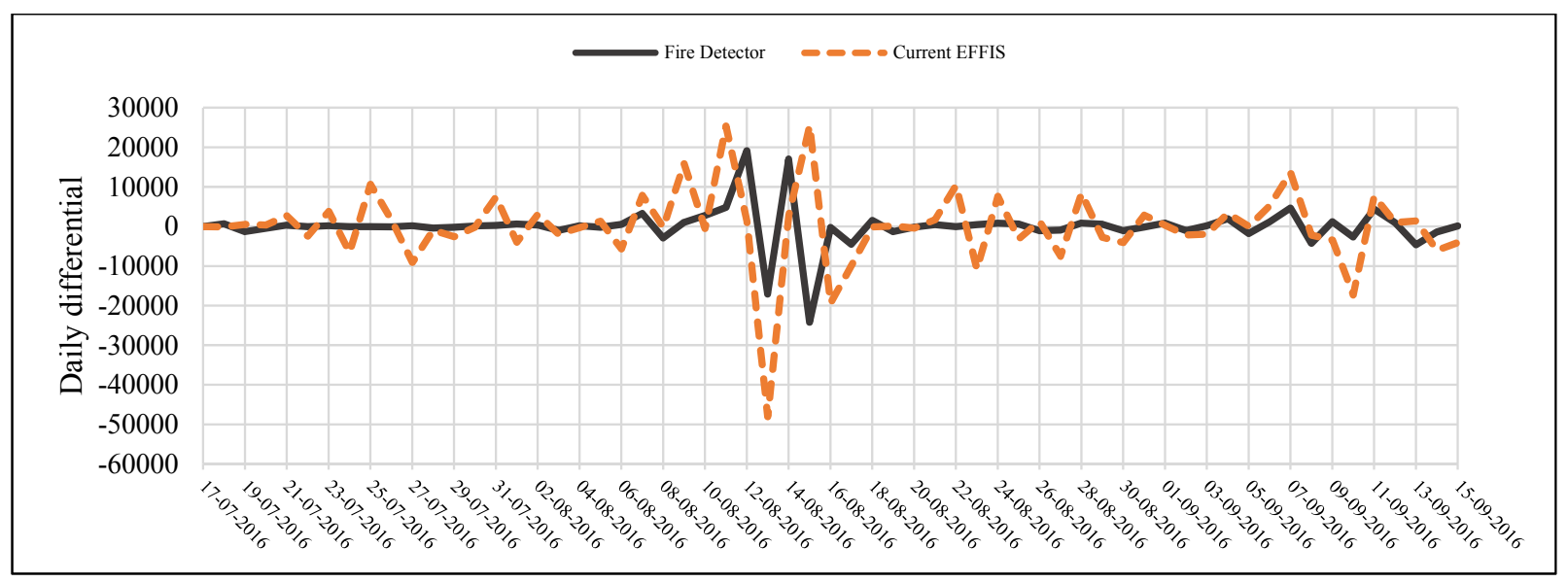

Figure 9. Daily differential burnt area.

Moreover, when comparing the burnt area in Figure 7, is clear that the fire detection system is underestimating the total burnt area. As can be seen in Figure 8, there is an daily error that affects to the accumulated burnt area. In addition, since some fires are not even detected during august the difference of the burnt area between both methods increases.

Finally, the daily differential of the burnt area, Figure 9, depicts a similar behavior in both methods. The proposed approach depicts a considerable amount of daily-burnt area omitted and a similar trend during the most intense days of the fire season.

In terms of burnt area, is mandatory to use some additional method to increase the accuracy of the estimated burnt area. The thermal anomalies is crucial tool for fire detection. Nevertheless, it has limitations using this approach for forest fire mapping. For this reason, this work proposes the use of the fire detections to use satellite imagery only over the fire extents for a better-burnt area estimation.

\section{EXPLOITING THE FIRE DETECTION FOR BURNT AREA MAPPING}

This section describes how the amount of requested and processed data from Sentinel-2 can be reduced using the fire extents of each fire could be used to request and process images from Sentinel-2 reducing the amount of data requested and processed.

As soon as the fire detector creates the list of fire objects, each non-active fire is grouped by UTM zone and the complementary tiling grid of Sentinel-2 [16], where only the desired bands of such tiles are downloaded. As first approach only band 8 and 12 are used to compute the differenced normalized burnt ratio (dNBR) [17] that compares images before and after the fire event. During the request, an image fetching method is developed to discard not useful imagery. For instance, such images where the fire extents falls in nodata area, or the cloud coverage over the estimated area is high are discarded and other date is requested. Therefore, the complementary module of the fire detector requests and processes each fire extent using several parallel processes in a single machine.

Figure 10 depicts the potential of this approach showing how this method is feasible to process satellite imagery using minimal resources automatically focusing on the fire extents. 


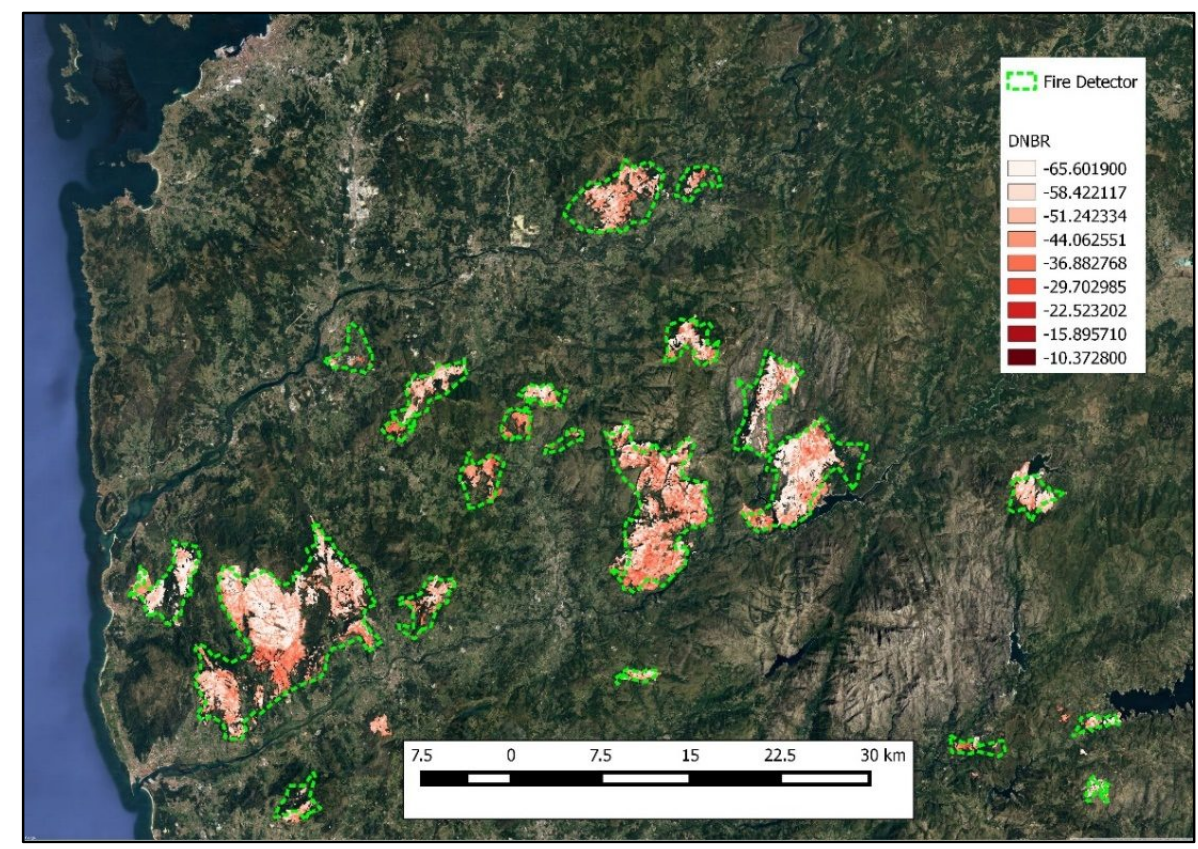

Figure 10. dNBR computed from Sentinel-2 computing only the extents produced by the fire detector based on thermal anomalies. North Portugal during fire season 2016.

Also, some other techniques for fire mapping could be added to increase the accuracy of the fire mapping like the semiautomatic method proposed by National Observatory of Forest Fires (NOFFI) [18]. For instance, normalized vegetation index (NDVI) could be computed to carry out an auto segmentation at low resolution, using a single image or the difference of the NDVI. Afterwards, a classification of each pixel is done at full resolution. Finally, those areas which match better regarding the rest of burnt areas estimations would be selected. An example of auto segmentation is shown in Figure 11.

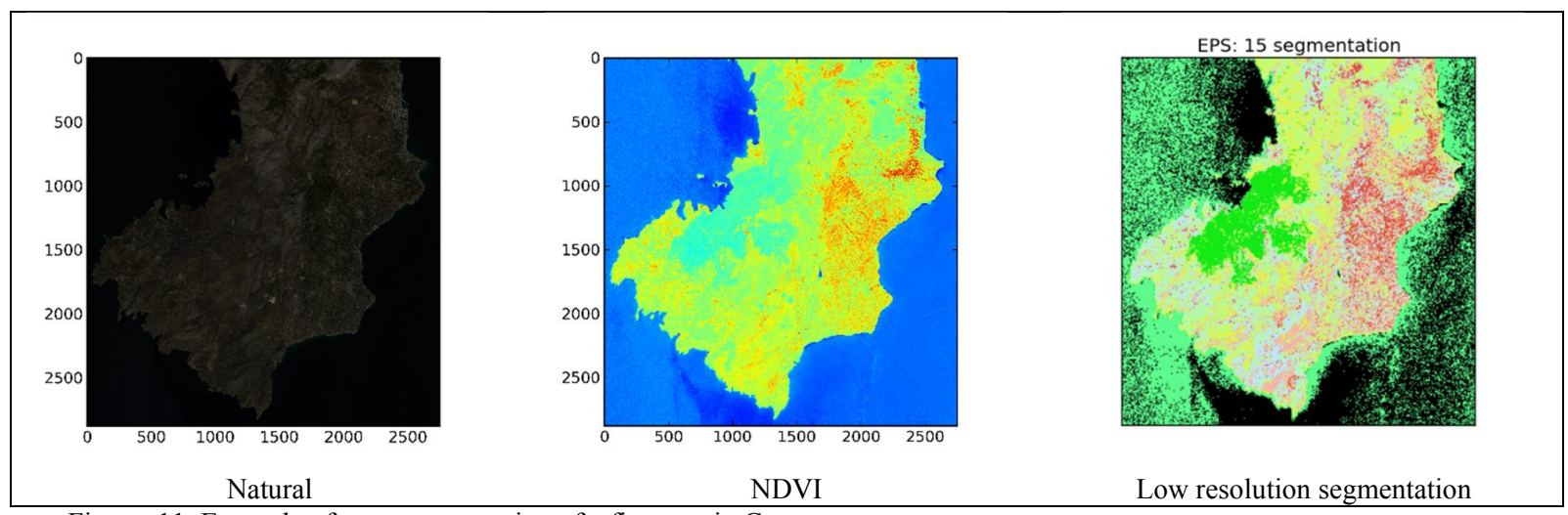

Figure 11. Example of auto segmentation of a fire case in Greece.

Several image-processing techniques could be applied since the fire extents are not a high workload. Additionally, the computation could be easily distributed to run in parallel environments with a final check step for those fires that are near enough to create artifacts between overlapping extents. This will correct the burnt scars that have been previously estimated using the alpha shape for each cluster of thermal anomalies. 


\section{CONCLUSIONS}

This work proposes a method for burnt area mapping combining different sources of information. The initial method detects the fires reducing the number of false positives and estimates a potential burnt area computing a convex hull applying an alpha shape. Although the fire detector could assess the task of focus on the areas where there is fire, the results show that could not be used as a generalized method to compute burnt areas. However, it could be applied as a rapid mapping estimator when dealing with big fires. The main reason of this drawback is the spatial resolution of the thermal anomalies. In fact, this approach has been used to estimate the burnt area of big fires with a good performance. Therefore, this approach uses a very low computational cost providing a potential burnt area with a few hours of delay since the data acquisition of the sensors.

In addition, the fire detector results could be considered as a set of regions of interest, extents to focus the processing combining other sources. In this work, Sentinel-2 images are used to complement the initial estimation. This combination allows to download and process the minimum data required to improve the accuracy using other burnt scar mapping approaches.

The method could be globally applied replacing the CLC2012 by a global forest cover layer. Besides, to avoid the false positives, different fire behavior parameters for each zone should be defined given the different behavior of the fire in different areas.

This work could evolve to a more solid and validated system for fire monitoring. The potential use of the results could be relevant for emergency management departments and for the research community a middle-long term. One of the main challenges in fire modelling is to obtain a good dataset describing the evolution of the fire. Combining as many sources as possible and processing all of them, it would help to create such datasets and help to develop and validate forest fire spread models.

\section{REFERENCES}

[1] L. R. Iverson, T. F. Hutchinson, A. M. Prasad and M. P. Peters, "Thinning, fire, and oak regeneration across a heterogeneous landscape in the eastern U.S.: 7-year results," Forest Ecology and Management, vol. 255, pp. 30353050, 2008.

[2] I. Verkaik and J. M. Espelta, "Post-fire regeneration thinning, cone production, serotiny and regeneration age in Pinus halepensis," Forest Ecology and Management, vol. 231, pp. 155-163, 2006.

[3] J. L. Coen, M. Cameron, J. Michalakes, E. G. Patton, P. J. Riggan and K. M. Yedinak, "WRF-Fire: coupled weather--wildland fire modeling with the weather research and forecasting model," Journal of Applied Meteorology and Climatology, vol. 52, pp. 16-38, 2013.

[4] T. Artés, A. Cencerrado, A. Cortés and T. Margalef, "Real-time genetic spatial optimization to improve forest fire spread forecasting in high-performance computing environments," International Journal of Geographical Information Science, vol. 30, pp. 594-611, 2016.

[5] T. Srivas, T. Artes, A. de Callafon and Raymond and I. Altintas, "Wildfire Spread Prediction and Assimilation for FARSITE Using Ensemble Kalman Filtering," Procedia Computer Science, vol. 80, pp. 897-908, 2016.

[6] J. Mandel, J. D. Beezley, A. K. Kochanski, V. Y. Kondratenko and M. Kim, "Assimilation of perimeter data and coupling with fuel moisture in a wildland fire--atmosphere DDDAS," Procedia Computer Science, vol. 9, pp. 1100-1109, 2012.

[7] I. Altintas, J. Block, R. De Callafon, D. Crawl, C. Cowart, A. Gupta, M. Nguyen, H.-W. Braun, J. Schulze, M. Gollner and others, "Towards an integrated cyberinfrastructure for scalable data-driven monitoring, dynamic prediction and resilience of wildfires," Procedia Computer Science, vol. 51, pp. 1633-1642, 2015.

[8] N. Dobrinkova, G. Jordanov and J. Mandel, "WRF-fire applied in Bulgaria," in International Conference on Numerical Methods and Applications, 2010. 
[9] I. A. Csiszar, J. T. Morisette and L. Giglio, "Validation of active fire detection from moderate-resolution satellite sensors: the MODIS example in northern Eurasia," IEEE Transactions on Geoscience and Remote Sensing, vol. 44, pp. 1757-1764, 2006.

[10] W. Schroeder, P. Oliva, L. Giglio and I. A. Csiszar, "The New VIIRS 375m active fire detection data product: Algorithm description and initial assessment," Remote Sensing of Environment, vol. 143, pp. 85-96, 2014.

[11] C. L. Cover, "Corine Land Cover," European Environment Agency, Copenhagen, 2000.

[12] T. Artés, Multi-core hybrid architectures applied to forest fire spread prediction, Barcelona: Universitat Autònoma de Barcelona, 2015.

[13] M. Ester, H.-P. Kriegel, J. Sander, X. Xu and others, "A density-based algorithm for discovering clusters in large spatial databases with noise.," in Kdd, 1996.

[14] F. Pedregosa, G. Varoquaux, A. Gramfort, V. Michel, B. Thirion, O. Grisel, M. Blondel, P. Prettenhofer, R. Weiss, V. Dubourg and others, "Scikit-learn: Machine learning in Python," Journal of Machine Learning Research, vol. 12, pp. 2825-2830, 2011.

[15] N. Chiaraviglio, T. Artés, R. Bocca, J. López, A. Gentile, J. S. M. Ayanz, A. Cortés and T. Margalef, "Automatic fire perimeter determination using MODIS hotspots information," in e-Science (e-Science), 2016 IEEE 12th International Conference on, 2016.

[16] M. Drusch, U. Del Bello, S. Carlier, O. Colin, V. Fernandez, F. Gascon, B. Hoersch, C. Isola, P. Laberinti, P. Martimort and others, "Sentinel-2: ESAś optical high-resolution mission for GMES operational services," Remote Sensing of Environment, vol. 120, pp. 25-36, 2012.

[17] A. E. Cocke, P. Z. Fulé and J. E. Crouse, "Comparison of burn severity assessments using Differenced Normalized Burn Ratio and ground data," International Journal of Wildland Fire, vol. 14, pp. 189-198, 2005.

[18] M. Tompoulidou, A. Stefanidou, D. Grigoriadis, E. Dragozi, D. Stavrakoudis and I. Z. Gitas, "The Greek National Observatory of Forest Fires (NOFFi)," in Fourth International Conference on Remote Sensing and Geoinformation of the Environment, 2016.

[19] J. a. B. J. D. a. K. A. K. a. K. V. Y. a. K. M. Mandel, "Assimilation of perimeter data and coupling with fuel moisture in a wildland fire--atmosphere DDDAS," in Procedia Computer Science, 2012. 